\title{
BMJ Open Comparative efficacy and side-effect profile of ketamine and esketamine in the treatment of unipolar and bipolar depression: protocol for a systematic review and network meta-analysis
}

\author{
Zhiqing Zhan, ${ }^{1}$ Xichao Wang, ${ }^{2}$ Qing Chen, ${ }^{2}$ Zhidai Xiao, ${ }^{2}$ Bin Zhang (1) ${ }^{2}$
}

To cite: Zhan Z, Wang $X$, Chen $Q$, et al. Comparative efficacy and side-effect profile of ketamine and esketamine in the treatment of unipolar and bipolar depression: protocol for a systematic review and network meta-analysis. BMJ Open 2021;11:e043457. doi:10.1136/ bmjopen-2020-043457

- Prepublication history for this paper is available online. To view these files, please visit the journal online (http://dx.doi. org/10.1136/bmjopen-2020043457).

Received 05 August 2020 Revised 29 January 2021 Accepted 01 February 2021

Check for updates

(c) Author(s) (or their employer(s)) 2021. Re-use permitted under CC BY-NC. No commercial re-use. See rights and permissions. Published by BMJ.

${ }^{1}$ The Third Clinical College, Guangzhou Medical University, Guangzhou, China

${ }^{2}$ PsyNI Lab, The Affiliated Brain Hospital of Guangzhou Medical University, Guangzhou, China

Correspondence to

Dr Bin Zhang;

zhang.bin845@foxmail.com

\section{ABSTRACT}

Introduction Despite a range of antidepressant drugs and therapies, approximately one-third of patients fail to achieve meaningful recovery, prompting the urgent need for more effective treatment for depression. Several open-label studies randomised controlled trials (RCTs) and meta-analyses have been conducted to confirm the therapeutic efficacy and side effects of ketamine and esketamine. Esketamine is (S)- enantiomer of ketamine; however, there is limited evidence comparing esketamine and ketamine in treating unipolar and bipolar depression have been published so far.

Methods and analysis We will include all doubleblind RCTs comparing efficacy and side-effect profile of ketamine and esketamine in the treatment of unipolar and bipolar depression. Our primary outcomes will be study-defined response at endpoint assessment; dropouts due to adverse events and other adverse drug reactions. Published studies will be retrieved through relevant database searches. Reference selection and data extraction will be independently completed by two investigators, resolving inconsistencies by consensus or a discussion with the third investigator. For each outcome, we will undertake a network meta-analysis to synthesise all evidence. Local and global methods will be used to evaluate consistency. We will assess the quality of evidence contributing to network estimates with the Confidence in Network Meta-Analysis web application. Ethics and dissemination This work does not require ethics approval as it will be based on published studies. This review will be published in peer-reviewed journals. PROSPERO registration number CRD42020201559.

\section{INTRODUCTION}

Depression is a mood disorder, which is characterised by feeling of sadness, helplessness and worthlessness as well as psychological symptoms including lack of energy, sleep cycle disturbance and appetite changes. ${ }^{1}$ It is the leading cause of disability in the world, affecting nearly 300 million individuals globally. ${ }^{23}$ Although depressive symptoms may be reduced within several weeks following the
Strengths and limitation of this study

- Supplied with advanced data analysing technologies, this systematic review and network meta-analysis would be the first to examine the comparative efficacy and side-effect profile of ketamine and esketamine in the treatment of unipolar and bipolar depressions.

- Qualitative evidence summary and network metaanalysis will address some highly relevant clinical questions in unipolar and bipolar depression. Subgroup analyses will further enhance the applicability of the results from this study.

- Only studies in English and Chinese will be included, which may cause language bias.

- There may be heterogeneity among the included studies due to the variety of participants' characteristics and study design.

initiation of conventional antidepressants, approximately one-third of patients fail to achieve meaningful recovery. ${ }^{4}$ Consequently, there is an ongoing search for more effective treatment for depression. ${ }^{5}$

Ketamine is a racemic mixture comprised of two enantiomers (R)-ketamine and (S)-ketamine and acts as an N-methyl-D-aspartate (NMDA) receptor antagonist. ${ }^{6}$ It has been found to have rapid antidepressant and antisuicidal effects at subanesthetic doses in several open-label studies randomised controlled trials (RCTs) and meta-analyses. ${ }^{7-9}$ However, the applicability of ketamine is limited in outpatient settings, for which it may require intravenous administration in the treatment of depression. ${ }^{10}$

Esketamine, the S(-)-enantiomer of ketamine, has a greater affinity with the NMDA receptor than the R(-)-enantiomer. ${ }^{11}$ To date, although the antisuicidal effects of esketamine have been inconsistent across 
several studies, $^{12} 13$ a recently published meta-analysis concluded that intranasal esketamine appears to have an ultrarapid antidepressant effect for major depressive disorder (MDD). ${ }^{14} \mathrm{~A}$ study also pointed out the rapid antidepressant response of intranasal esketamine over placebo started as early as within 2 hour, which is superior to that of single ketamine infusion for MDD. ${ }^{15}$

To date, there are several studies ${ }^{10} 1316-20$ evaluating the efficacy and side-effect profile of the enantiomers of ketamine, including esketamine, ${ }^{10} 13$ 16-19 the $\mathrm{S}$ (-)-enantiomer of ketamine and arketamine; ${ }^{20}$ the $\mathrm{R}$ (-)-enantiomer of ketamine; however, only one head-tohead study assessed the efficacy and safety of ketamine compared with esketamine. ${ }^{21}$ The population of this study was limited to patients with treatment-resistant depression; its sample size was relatively small. The evidences for ketamine comparing with esketamine in the treatment of unipolar and bipolar depression remain limited. Thus, a systematic review and meta-analysis will be performed to evaluate efficacy and side-effect profile of ketamine and esketamine in the treatment of unipolar and bipolar depressions.

\section{Objective}

To conduct a systematic review and network meta-analysis of RCTs evaluating the comparative efficacy and sideeffect profile of ketamine and esketamine in the treatment of unipolar and bipolar depressions.

\section{METHODS AND ANALYSIS}

Preferred Reporting Items for Systematic Reviews and Meta-Analyses Protocols will be set as a guide book for the protocol. ${ }^{22}$

\section{Patient and public involvement}

This work will be based on published studies; therefore, patient or public involvement will be not required. Relevant results will be published in peer-reviewed journals.

\section{Eligibility criteria}

Any studies not meeting following inclusion criteria will be excluded.

A. Participants: patients with unipolar and bipolar depressions according to any international diagnostic criteria.

B. Interventions: we considered studies examining any ketamine or esketamine as a standalone treatment or in combination with psychotropic medications or psychotherapies. Any route of administration is acceptable.

C. Comparators: placebo including inactive control (eg, saline) and active control (eg, other antidepressants or anxiolytics).

D. Outcome measures:

\section{The primary outcomes}

1. Study-defined response at endpoint assessment (eg, $\geq 50 \%$ reduction from baseline in Montgomery-Åsberg
Depression Rating Scale (MADRS) ${ }^{23}$ or Hamilton Depression Rating Scale (HAMD) ${ }^{24}$ and study-defined remission at endpoint assessment (eg, MADRS total score $\leq 12^{16}$ or 10$) .{ }^{10}$ When both the MADRS and HAMD were reported, only the MADRS will be used.

2. Dropouts due to adverse events (tolerability), measured as the proportion of patients who withdrew from the study, either during the acute-phase treatment or during the continuation/maintenance treatment, due to any adverse events (including specific adverse events and withdrawal symptoms).

3. Other adverse drug reactions.

\section{Secondary outcomes}

1. Study-defined response and remission at study-defined time points apart from treatment endpoint.

2. Severity of depressive symptoms as measured by standardised rating scales such as the MADRS. ${ }^{23}$

3. Suicide risk as measured by standardised rating scales, such as the Scale for Suicidal Ideations. ${ }^{25}$

4. Socio-occupational disability as measured by the Sheehan Disability Scale. ${ }^{26}$

\section{E. Study design}

We will include studies with double-blind RCTs focusing on the comparative efficacy and side-effect profile of ketamine and esketamine in the treatment of unipolar and bipolar depressions. Cross-over and cluster randomised trials will be included, while quasirandomised trials will be excluded. For cross-over studies, to address concerns around possible 'carry over' effects, we will use data from the precrossover phase. ${ }^{27}$ For studies that fail to report precrossover phase data, we will contact study authors to obtain this data.

\section{Searching strategy}

The relevant articles will be identified by searching the electronic bibliographic databases: PubMed, Web of Science, EMbase, Wanfang Data, China National Knowledge Infrastructure (CNKI). Medical subject headings will be used. The searching strategies of PubMed (English) and CNKI (Chinese) are shown in table 1 as examples to replicate for the other databases.

\section{Selection process}

We will use EndNote V.X7 software (Thomson Reuters, New York, USA) to collate search results and perform filtering. After removing duplicates, two independent reviewers will review the title and abstract of the identified studies and will exclude irrelevant parts. The full article will then be obtained and re-examined for more details to be included. Any conflict will be resolved through a discussion involving the third reviewer. This process will be completed by 25 February 2021 .

\section{Data collection}

The data will be extracted independently by three investigators, and when inconsistencies are encountered, they will be resolved by consensus or discussion with another 
Table 1 Searching strategy

\begin{tabular}{ll} 
1.1 PubMed searching strategy & $\mathbf{1 . 2}$ CNKI searching strategy \\
\hline \#1 depression (MeSH Terms) & \#1抑有症 (主题词) \\
\#2 depression (All Fields) & \#2抑郁(主题词) \\
\#3 depress (All Fields) & \#3单相抑郁症 (主题词 ) \\
\#4 melancholia (All Fields) & \#4忧郁症 (主题词 ) \\
\#5 bipolar disorder (All Fields) & \#5抑郁综合征(主题词) \\
\#6 bipolar spectrum (All Fields) & \#6双相障碍(主题词) \\
\#7\#1OR \#2 OR \#3 OR \#4 OR \#5OR \#6 & \#7 \#1 OR \#2 OR \#3 OR \#4 OR \#5 OR \#6 \\
\#8 Ketamine (MeSH Terms) & \#8氯胺酮 (主题词) \\
\#9 Esketamine (All Fields) & \#9艾氯胺酮(主题词) \\
\#10 \#8 OR \#9 & \#10艾司氯胺酮(主题词) \\
\#11 Final search terms: \#7 AND \#10 \#11\#8 OR \#9OR \#10 & \#12 Final search terms: \#7 AND \#11
\end{tabular}

investigator. Information extracted will include publication information (eg, title, first author, publishing time, unit, country and funding support), details of interventions (eg, ketamine or esketamine dose and route of administration, concomitant medications dose and route of administration), details of comparator (eg, names of control measures, dose and route of administration), details of methodology (eg, participants, sample size, diagnostic criteria, demographic characteristics, data analysis strategies, clinical assessment and clinical variables) and outcome measures. If necessary, we will contact the first/corresponding author by e-mail to obtain additional information when the meta-analysis data were not reported.

\section{Quality assessment}

The risk of bias of individual trials and the certainty of evidence contributing to network estimates of the primary outcomes will be assessed by the Cochrane Collaboration Risk of Bias Tool (http://handbook-5-1.cochrane.org/), including the following criteria:

1. random sequence generation or allocation concealment.

2. Blinding of outcome assessment.

3. Selective outcome reporting.

4. Incomplete data assessment.

Two reviewers (ZX and XW) will carry out the quality assessment independently. Any disagreement will be resolved by discussion with the third reviewers $(\mathrm{ZZ})$.

GRADEPro software (V.3.2 for Windows) will be used to evaluate the overall quality of the evidence.

\section{Data synthesis}

We will generate descriptive statistics for the trial, and study population characteristics across all eligible trials, describing the types of comparisons and some important variables, either clinical or methodological (such as year of publication, age, severity of illness, sponsorship and clinical setting). We will draw the network diagram to graphically present the available evidence.

\section{Assessment of heterogeneity (pairwise meta-analysis)}

We will conduct a pairwise meta-analysis comparing ketamine and esketamine versus placebo to investigate the effects of antidepressants in the treatment by visually inspecting the forest plots. For this analysis, heterogeneity will be assessed using Cochran $\mathrm{X}^{2}$ test and further quantified using $\mathrm{I}^{2}$ to decide which effect models are used for meta-analysis. A fixed-effect model will be used to analyse the data if there is no evidence of heterogeneity $(\mathrm{p} \geq 0.1$, $\left.\mathrm{I}^{2} \leq 50 \%\right)$. A random-effect model will be used if heterogeneity exists $\left(\mathrm{p}<0.1, \mathrm{I}^{2}>50 \%\right)$ and the possible causes from both clinical and methodological will be searched. In this analysis, we will consider all primary and secondary outcomes. We will assess heterogeneity using the tool described in the Cochrane Collaboration Handbook as a reference guide (http://handbook-5-1.cochrane.org/).

\section{Assessment of the transitivity assumption of network meta- analysis}

To assess whether the distribution of potential effect modifiers is balanced across treatment comparisons, we will check for significant differences in the distribution of the following factors among different comparisons: severity of depression before randomisation, age, average number of previous episodes and clinical and demographic characteristics such as age, gender, dose and severity of symptoms. We will group studies by treatment comparisons and obtain descriptive statistics regarding these important covariates. If significant discrepancies are found in the corresponding distributions, our network meta-analysis will be limited to studies which are considered sufficiently similar.

\section{Network meta-analyses}

Network meta-analysis will be performed to synthesise the evidence if there is no evidence against the transitivity 
assumption. We will use a random-effect network metaanalysis model ${ }^{28}$ fit in a frequentist setting, assuming a common heterogeneity parameter across all treatment comparisons. We will present the results including all estimated treatment effects and the corresponding $95 \%$ CIs. For the assessment of the extent of heterogeneity, the estimated value for the heterogeneity SD and the corresponding empirical distributions will be compared in each outcome. Furthermore, we will calculate the ranking probabilities for all treatments (including all available single drugs and combinations) of being at each possible rank for each intervention, using the surface under the cumulative ranking. The network meta-analyses (NMA) will be performed by the package 'netmeta' (V.0.5-0) in R (V.3.0.2, The R Foundation for Statistical Computing). We will use package 'igraph,29 and package 'ggplot2',30 to visualise the networks and the distribution of ranking probability distribution.

\section{Assessment of inconsistency}

Another key assumption for performing an NMA is the consistency in the agreement between the direct and indirect sources of the network. A common evaluation method of inconsistency is hypothesis test. Since our network meta-analysis focuses on two interventions, ketamine and esketamine, we will use the Z-test to assess the consistency. The Z-test ${ }^{31}$ is often used to evaluate the consistency of a network meta-analysis of two-arm RCTs. For continuous variables, we will use the difference of the mean difference (the difference between the direct and indirect comparison results) and its $95 \%$ CI to assess the inconsistency. The $\mathrm{p}$ value will be calculated to assess whether there is statistical agreement between direct and indirect evidence on the same comparisons. $\mathrm{p}<0.05$ will be considered to be statistically significant. For continuous variables, we will calculate directly according to the result (mean difference).

\section{Sensitivity analyses and subgroup analyses}

We will conduct a sensitivity analysis to verify the robustness of the study conclusions and assess the impact of methodological quality, study design, sample size and the effect of missing data as well as the analysis methods on the result of this review. ${ }^{32}$ Studies meet the following criterion will be analysed to evaluate the sensitivity of our conclusions:

1. Studies with balanced doses in all arms.

2. Studies with low risk of bias.

3. Head-to-head studies.

We will also conduct the network meta-regression on primary outcomes for the:

1. Sex ratio.

2. The types of depression.

3. Duration treatment.

4. Depressive severity at baseline.

5. Dosing schedule. We excluded combinations of drugs from the network meta-regression because we cannot be sure whether some combinations can affect the dose effects.

\section{Assessment of publication bias and small study effect}

In order to minimise the potential impact due to publication bias or other reporting biases, we will conduct a comprehensive retrieval and choose the eligible studies totally by strict standard. Funnel plots will be used to assess the existence of small study effects and publication biases. ${ }^{33}$ We will use the Harbord test ${ }^{34}$ to formally test for asymmetries in the funnel plots. If we identify an important association of the reported effect with the trials' precision, we will try to adjust for it in a sensitivity analysis. Then a network meta-regression with the trial precision as a study-level covariate will be performed. If the results indicating that there is strong evidence of small study effects or publication bias, we will clearly report it and interpret our results cautiously.

\section{Ethics and dissemination}

Ethical approval is not required as primary data will not be collected. This review will be published in a peerreviewed journal.

Contributors BZ was responsible for this study. BZ and ZZ conceived and designed the study. ZZ, XW, ZX and QC participated in drafting the protocol and preparing the manuscript. All authors read and approved the final manuscript.

Funding National Natural Science Foundation of China (grant number: 81801345). Competing interests None declared.

Patient and public involvement Patients and/or the public were not involved in the design, or conduct, or reporting, or dissemination plans of this research.

Patient consent for publication Not required.

Provenance and peer review Not commissioned; externally peer reviewed.

Open access This is an open access article distributed in accordance with the Creative Commons Attribution Non Commercial (CC BY-NC 4.0) license, which permits others to distribute, remix, adapt, build upon this work non-commercially, and license their derivative works on different terms, provided the original work is properly cited, appropriate credit is given, any changes made indicated, and the use is non-commercial. See: http://creativecommons.org/licenses/by-nc/4.0/.

ORCID iD

Bin Zhang http://orcid.org/0000-0002-9280-8247

\section{REFERENCES}

1 Valentine, Barbara Ret al. American psychological association. College \& Research Libraries News 2001;62:215.

2 Charlson F, van Ommeren M, Flaxman A, et al. New WHO prevalence estimates of mental disorders in conflict settings: a systematic review and meta-analysis. Lancet 2019;394:240-8.

3 Herrman $\mathrm{H}$, Kieling C, McGorry P, et al. Reducing the global burden of depression: a Lancet-World psychiatric association Commission. Lancet 2019;393:e42-3.

4 Corriger A, Pickering G. Ketamine and depression: a narrative review. Drug Des Devel Ther 2019;13:3051-67.

5 Shah AA. Novel approaches for managing treatment-resistant depression. Psychiatr Ann 2016;46:220-2.

6 Jelen LA, Young AH, Stone JM. Ketamine: a tale of two enantiomers. J Psychopharmacol 2021;35:269881120959644.

7 aan het Rot M, Collins KA, Murrough JW, et al. Safety and efficacy of repeated-dose intravenous ketamine for treatment-resistant depression. Biol Psychiatry 2010;67:139-45.

8 Zheng W, Zhou YL, Liu WJ. Rapid and longer-term antidepressant effects of repeated-dose in travenous ketamine for patients with unipolar and bipolar depression. J Psychopharmacol 2018;106:61-8. 
9 Zheng W, Zhou Y-L, Liu W-J, et al. Investigation of medical effect of multiple ketamine infusions on patients with major depressive disorder. J Psychopharmacol 2019;33:494-501.

10 Daly EJ, Singh JB, Fedgchin M, et al. Efficacy and safety of intranasal Esketamine adjunctive to oral antidepressant therapy in treatment-resistant depression: a randomized clinical trial. JAMA Psychiatry 2018;75:139-48.

11 Pochwat B, Nowak G, Szewczyk B. An update on NMDA antagonists in depression. Expert Rev Neurother 2019;19:1055-67.

12 Canuso CM, Singh JB, Fedgchin M, et al. Efficacy and safety of intranasal Esketamine for the rapid reduction of symptoms of depression and suicidality in patients at imminent risk for suicide: results of a double-blind, randomized, placebo-controlled study. Focus 2019;17:55-65.

13 Popova V, Daly EJ, Trivedi M, et al. Efficacy and safety of flexibly dosed Esketamine nasal spray combined with a newly initiated ora antidepressant in treatment-resistant depression: a randomized double-blind active-controlled study. AJP 2019;176:428-38.

14 Zheng W, Cai D-B, Xiang Y-Q, et al. Adjunctive intranasal esketamine for major depressive disorder: a systematic review of randomized double-blind controlled-placebo studies. J Affect Disord 2020;265:63-70

15 Kishimoto T, Chawla JM, Hagi K, et al. Single-Dose infusion ketamine and non-ketamine $\mathrm{N}$-methyl-D-aspartate receptor antagonists for unipolar and bipolar depression: a meta-analysis of efficacy, safety and time trajectories. Psychol Med 2016;46:1459-72.

16 Canuso CM, Singh JB, Fedgchin M, et al. Efficacy and safety of intranasal Esketamine for the rapid reduction of symptoms of depression and suicidality in patients at imminent risk for suicide: results of a double-blind, randomized, placebo-controlled study. Am J Psychiatry 2018;175:620-30.

17 Kryst J, Kawalec P, Pilc A. Efficacy and safety of intranasal esketamine for the treatment of major depressive disorder. Expert Opin Pharmacother 2020;21:9-20.

18 Fedgchin M, Trivedi M, Daly EJ, et al. Efficacy and safety of fixed-dose Esketamine nasal spray combined with a new ora antidepressant in treatment-resistant depression: results of a randomized, double-blind, active-controlled study (TRANSFORM-1). Int J Neuropsychopharmacol 2019;22:616-30.

19 Fu D-J, Ionescu DF, Li X, et al. Esketamine nasal spray for rapid reduction of major depressive disorder symptoms in patients who have active suicidal ideation with intent: double-blind, randomized study (ASPIRE I). J Clin Psychiatry 2020;81:19m13191.
20 Leal GC, Bandeira ID, Correia-Melo FS, et al. Intravenous arketamine for treatment-resistant depression: open-label pilot study. Eur Arch Psychiatry Clin Neurosci 2020. doi:10.1007/s00406-020-01110-5. [Epub ahead of print: 20 Feb 2020].

21 Correia-Melo FS, Leal GC, Vieira F, et al. Efficacy and safety of adjunctive therapy using esketamine or racemic ketamine for adult treatment-resistant depression: a randomized, double-blind, noninferiority study. J Affect Disord 2020;264:527-34.

22 Moher D, Shamseer L, Clarke M, et al. Preferred reporting items for systematic review and meta-analysis protocols (PRISMA-P) 2015 statement. Syst Rev 2015;4:g7647.

23 Montgomery SA, Asberg M. A new depression scale designed to be sensitive to change. Br J Psychiatry 1979;134:382-9.

24 Hamilton M. A rating scale for depression. J Neurol Neurosurg Psychiatry 1960;23:56-62.

25 Beck AT, Kovacs M, Weissman A. Assessment of suicidal intention: the scale for suicide ideation. J Consult Clin Psychol 1979;47:343-52.

26 Leon AC, Olfson M, Portera L, et al. Assessing psychiatric impairment in primary care with the Sheehan disability scale. Int $J$ Psychiatry Med 1997;27:93-105.

27 Furukawa TA, Salanti G, Atkinson LZ, et al. Comparative efficacy and acceptability of first-generation and second-generation antidepressants in the acute treatment of major depression: protocol for a network meta-analysis. BMJ Open 2016;6:e010919.

28 Rücker G, Schwarzer G, Gerta R, Guido S. Reduce dimension or reduce weights? comparing two approaches to multi-arm studies in network meta-analysis. Stat Med 2014;33:4353-69.

29 Csardi G, Nepusz T. The igraph software package for complex network research. Inter J Complex Syst 2006;1695:1-9.

30 Wickham H. Ggplot2: elegant graphics for data analysis. Journal of the International Biometric Society 2011;67:678-9.

31 Song F, Xiong T, Parekh-Bhurke S, et al. Inconsistency between direct and indirect comparisons of competing interventions: metaepidemiological study. BMJ 2011;343:d4909.

32 Egger M, Davey Smith G, Schneider M, et al. Bias in meta-analysis detected by a simple, graphical test. BMJ 1997;315:629-34.

33 Peters JL, Sutton AJ, Jones DR, et al. Contour-enhanced metaanalysis funnel plots help distinguish publication bias from other causes of asymmetry. J Clin Epidemiol 2008;61:991-6.

34 Harbord RM, Egger M, Sterne JAC. A modified test for small-study effects in meta-analyses of controlled trials with binary endpoints. Stat Med 2006;25:3443-57. 